\title{
Emittance of a photocathode: Effects of temperature and field
}

\author{
Kevin L. Jensen \\ Naval Research Laboratory, Washington, D.C. 20375-5347, USA \\ P. G. O'Shea and D. W. Feldman \\ University of Maryland, College Park, Maryland 20742-3511, USA \\ (Received 22 February 2010; published 5 August 2010)
}

\begin{abstract}
The emittance of a photocathode is evaluated using a distribution function ("Moments") approach to calculate the moments of the momentum. The effects of temperature and field, which affect the electron distribution and transmission probability, respectively, of electrons incident on the surface barrier, are found. The resulting formulations of emittance are compared to the asymptotic limit found by D.H. Dowell and J.F. Schmerge [Phys. Rev. ST Accel. Beams 12, 074201 (2009)], and their formulation is shown to be more generally applicable than the approximations within it would indicate for metals. The methodology is extended to develop an asymptotic emittance estimate for semiconductor photocathodes.

DOI: 10.1103/PhysRevSTAB.13.080704

PACS numbers: 52.59.Wd, 07.77.Ka, 85.60.Ha, 41.60.Cr
\end{abstract}

\section{INTRODUCTION}

The emittance of a photocathode is evaluated using a distribution function approach to calculate the moments of the momentum: it therefore accounts for material properties (e.g., work function and electron affinity) as well as the effects of temperature and applied field. Intense electron beams for high power and short wavelength free-electron lasers entail restrictions on the tolerable emittance from the electron source, typically a photocathode, to enable very short bunches of a desired shape [1]. Models of emittance share a methodology with the calculation of quantum efficiency, or QE [2]. In the distribution function approach used here, QE is proportional to the first moment of the longitudinal momentum $\left\langle k_{z}^{1}\right\rangle$, whereas emittance considers the square of the transverse momentum component $\left\langle k_{\rho}^{2}\right\rangle$. The Moments approach was the basis for the evaluation of emittance of a photocathode in earlier treatments [3-5]. Differences between it and the Spicer three-step model employed by Dowell and Schmerge (DS) were suggested to be due to differences in their respective approximations, but Dowell and Schmerge demonstrated that the difference instead was due to the neglect of the photon energy contribution to the transverse momentum in the Moments approach: when included, the two formulations agree in their asymptotic limits.

Given the equivalence of the Moments approach and the DS-Spicer approach, the present work reconstructs the Moments formulation without making the approximations leading to the elegant and simple asymptotic limit. These approximations are (i) the dominance of forward (into the surface barrier) transport or, alternately, the neglect of the angular dependence of the scattering probability, (ii) the energy independence of the mean free path, (iii) the stepfunction nature of the transmission probability, (iv) the temperature independence of all factors and, in particular, the zero temperature limit of the Fermi-Dirac distribution that characterizes electrons in metals, (v) the effect of electric field manifesting itself only in the Schottky barrier lowering factor $\sqrt{4 Q F}$ rather than in the transmission probability, and (vi) the presumed closeness of the photon energy $\hbar \omega$ to the work function $\Phi$. The nomenclature is as given in Table I. It shall be shown that the DS formula of the emittance of a metal photocathode remains good even when all other complications (in spite of their complexity) are considered, and over a larger range of values than otherwise expected. The methodology is then used to develop expressions for the emittance of semiconductor photocathodes, for which such approximations have greater consequence: a similar, but not as simple, formulation is found that shows greater differences with the numerically evaluated moments than is the case for metals.

\section{ZEROTH ORDER MOMENTS APPROXIMATION}

In terms of the conjugate position and momentum terms $x$ and $k_{x}$, respectively, the normalized rms emittance is given by [6]

$$
\varepsilon_{n, \mathrm{rms}}=\frac{\hbar}{m c} \sqrt{\left\langle x^{2}\right\rangle\left\langle k_{x}^{2}\right\rangle-\left\langle x k_{x}\right\rangle^{2}} .
$$

It is often the case that the cross term $\left\langle x k_{x}\right\rangle^{2}$ is negligible by comparison to the term $\left\langle x^{2}\right\rangle\left\langle k_{x}^{2}\right\rangle$ (but not always, as in the case of field emitters [7]), and that approximation holds here. For uniform emission, $\left\langle x^{2}\right\rangle=\left\langle\rho^{2}\right\rangle / 2=\rho_{c}^{2} / 2$, where $\rho_{c}$ is the radius of the emission area. As noted by Dowell and Schmerge, considerations of emittance therefore revolve around the behavior of $\left\langle k_{x}^{2}\right\rangle=\left\langle k_{\rho}^{2}\right\rangle / 2$. Moments are weighted averages over the distribution $f(\mathbf{r}, \mathbf{k})$ of emitted 
TABLE I. Parameters and representative values.

\begin{tabular}{lccc}
\hline \hline Symbol & Definition & Value & Unit \\
\hline$m$ & Electron mass (vacuum) & 510999 & $\mathrm{eV} / \mathrm{c}^{2}$ \\
$c$ & Speed of light & 299.792 & $\mathrm{~nm} / \mathrm{fs}$ \\
$\hbar$ & Planck's constant & 0.658212 & $\mathrm{eV} \mathrm{fs}$ \\
$\alpha_{\mathrm{fs}}$ & Fine structure constant & $1 / 137.036$ & $\cdots$ \\
$Q$ & $\alpha_{\mathrm{fs}} \hbar c / 4$ & 0.359991 & $\mathrm{eV} \mathrm{nm}$ \\
$T$ & Temperature & 300 & $\mathrm{~K}$ \\
$F$ & Electron charge $\times$ electric field & 0.1 & $\mathrm{eV} / \mathrm{nm}$ \\
$\Phi$ & Work function & $4.5(\mathrm{Cu}), 4.52(\mathrm{Ag})$ & $\mathrm{eV}$ \\
$\mu$ & Chemical potential & $7(\mathrm{Cu}), 5.48(\mathrm{Ag})$ & $\mathrm{eV}$ \\
$k_{F}$ & $(2 m \mu)^{1 / 2} / \hbar$ & $\ldots$ & $1 / \mathrm{nm}$ \\
$\hbar \omega$ & Photon energy & 4.661 & $\mathrm{eV}$ \\
$\rho_{c}$ & Laser illumination radius & 1 & $\mathrm{~mm}$ \\
$\delta_{\omega}$ & Laser penetration depth & 12 & $\mathrm{~nm}$ \\
$E_{a}$ & Electron affinity & $0.3\left(\mathrm{Cs}_{3} \mathrm{Sb}\right), 0.2\left(\mathrm{Cs}_{2} \mathrm{Te}\right)$ & $\mathrm{eV}$ \\
$E_{g}$ & Band gap & $1.6\left(\mathrm{Cs}_{3} \mathrm{Sb}\right), 3.5\left(\mathrm{Cs}_{2} \mathrm{Te}\right)$ & $\mathrm{eV}$ \\
\hline \hline
\end{tabular}

electrons and of the form [5]

$$
M_{n}\left(k_{\rho}\right) \propto \int k_{\rho}^{n} f(\mathbf{r}, \mathbf{k}) d \mathbf{r} d \mathbf{k},
$$

where the $n$ subscript on $M$ reflects the power $k$ is raised to-it is not related to the conventional $n$ subscript on $\varepsilon$ in Eq. (1) to indicate "normalized." The evaluation involves the ratio of the 2 nd to the 0th moment of the radial momentum: compared to the DS nomenclature, their "dimensionless rms transverse momentum" [Eq. (26) of [1] ] is equivalent to our $(\hbar / m c) \sqrt{M_{2}\left(k_{\rho}\right) / 2 M_{0}\left(k_{\rho}\right)}$-albeit that minor differences related to the transformation of the volume element in phase space being converted to an energy integration by assuming that $E_{k} \equiv(\hbar \mathbf{k})^{2} / 2 m$ occur-but to leading order such differences are negligible if the photon energy is comparable to the work function and slowly varying energy-dependent terms are evaluated at the Fermi level $\mu=\hbar^{2} k_{F}^{2} / 2 m$.

More importantly, as pointed out by Dowell and Schmerge, while the Moments approach references the momentum inside the metal, conservation of momentum in the transverse direction across the metal-vacuum barrier demands that $k_{\rho}$ outside be augmented by the photon energy via $k_{\rho}^{2} \rightarrow k_{\perp}^{2} \equiv k_{\rho}^{2}+k_{\omega}^{2}$, where $k_{\omega}^{2}=2 m \omega / \hbar^{2}$, and that emittance uses $\left\langle k_{\perp}^{2}\right\rangle$. The conservation of momentum argument of DS is equivalent to the demand of continuity of the wave function and its first derivative across the metal-vacuum interface. Therefore, earlier Moments treatments [specifically, Eq. (699) in Ref. [4] or Eq. (53) in Ref. [5] ] must replace $M_{n}\left(k_{\rho}\right)$ by $M_{n}\left(k_{\perp}\right)$ when applying the Moments approach to the emittance problem, otherwise the formula for emittance is a factor of $k_{F} / \sqrt{k_{F}^{2}+k_{\omega}^{2}}$ too small (e.g., $80 \%$ for $\mathrm{Cu}$ and UV light) compared to the DS asymptotic limit.
The evaluation of the Moments contains terms for absorption, transport to the surface, and emission over a surface barrier. For metals, the Moments are

$$
\begin{aligned}
M_{n}\left(k_{\perp}\right)= & (2 \pi)^{-3} \int d \mathbf{k}\left(k_{\perp}^{n}\right) D\left\{\left(E_{k}+\hbar \omega\right) \cos ^{2} \theta\right\} \\
& \times f_{\lambda}\left(\cos \theta, E_{k}+\hbar \omega\right) f_{\mathrm{FD}}(E) \\
& \times\left\{1-f_{\mathrm{FD}}\left(E_{k}+\hbar \omega\right)\right\},
\end{aligned}
$$

where transport is governed by the scattering factor $f_{\lambda}$, emission over the barrier is governed by the transmission probability $D$, and the absorption of the photon is governed by the Fermi-Dirac functions $f_{\mathrm{FD}}$ and is in proportion to the occupation of its initial state and dependent on whether its final state is occupied. Importantly, the scattering factor is given by $f_{\lambda}(\cos \theta, E)=\cos \theta /[\cos \theta+p(E)]$, where $p(E)$ is the ratio of the laser penetration depth $\delta_{\omega}$ to the distance between scattering events [i.e., the product of the velocity of the electron with its characteristic scattering time $\tau(E)]$.

If the Fermi-Dirac distribution and the transmission probability are taken as step functions (a zero temperature and field approximation for metals), if the window of energy allowed by the Fermi-Dirac functions the $p(E)$ term allows it to be replaced by $p_{o} \equiv p(\mu+\phi)$, and if the "forward dominated" approximation-that is, only those electrons pointing almost directly at the surface escape when the photon energy is near the effective work function $\phi$-is made, then the scattering factor is approximated by $f_{\lambda}(\cos \theta, E) \approx \cos \theta /\left(p_{o}+1\right)$ (though different than the equivalent DS approximation the leading order outcome is the same). The angular integration is then analytic and so 


$$
\begin{aligned}
M_{n}\left(k_{\perp}\right) \approx & \frac{1}{\left(8 \pi^{2}\right)}\left(\frac{2 m}{\hbar^{2}}\right)^{(n+3) / 2} \frac{1}{(n+2)\left(p_{o}+1\right)} \\
& \times \int_{\phi-\hbar \omega}^{0} \frac{\sqrt{\mu+s}(s+\hbar \omega-\phi)^{(n+2) / 2}}{\mu+s+\hbar \omega} d s,
\end{aligned}
$$

where $s$ is a dummy integration variable. For metals such as copper, $\mu$ is $7 \mathrm{eV}$, whereas $\hbar \omega-\phi$ is a fraction of an $\mathrm{eV}$ for wavelengths longer than UV. Therefore $\mu+s \approx \mu$ in the integrand, giving

$$
\begin{aligned}
M_{n}\left(k_{\perp}\right) \approx & \frac{\mu^{1 / 2}}{4 \pi^{2}(\mu+\hbar \omega)\left(p_{o}+1\right)(n+4)(n+2)} \\
& \times\left(\frac{2 m}{\hbar^{2}}\right)^{(n+3) / 2}(\hbar \omega-\phi)^{(n+2) / 2} .
\end{aligned}
$$

When applied to emittance it follows

$$
\varepsilon_{n, \mathrm{rms}}=\frac{\rho_{c}}{2} \frac{\hbar}{m c} \sqrt{\frac{M_{2}\left(k_{\perp}\right)}{2 M_{0}\left(k_{\perp}\right)}} \approx \frac{\rho_{c}}{2} \sqrt{\frac{\hbar \omega-\phi}{3 m c^{2}}} \equiv \varepsilon_{n, \mathrm{rms}}^{0},
$$

where the " 0 " superscript indicates the leading, or zeroth, order approximation, and is equivalent to Eq. (33) of Ref. [1]: what is now to be done is to not approximate the Fermi-Dirac distributions and the transmission probabilities as step functions, and retain the energy dependence of the integrand terms.

\section{CORRECTIONS TO THE ZEROTH ORDER APPROXIMATION}

Regarding the transmission probability as a step function and incorporating its effect on the angular integration part of $M_{n}$ results in integrals that can be analytically evaluated without recourse to the forward approximation or the relative constancy of the laser penetration depth $\delta_{\omega}(E)$ factor. Using the concise notation [see Eq. (697) of Ref. [4] or Eq. (62) of Ref. [5], but referred to as the function $G$ therein],

$$
\begin{aligned}
\eta_{0}(s)= & 1-s+p \ln \left[\frac{p+s}{p+1}\right] \\
\eta_{2}(s)= & \frac{1}{6}(1-s)\left[3 p(1+s)+2(2+s)(1-s)-6 p^{2}\right] \\
& +p\left(1-p^{2}\right) \ln \left[\frac{p+s}{p+1}\right]
\end{aligned}
$$

where the energy dependence of $s(E)=E+\hbar \omega$ and $p(E)=m \delta(\omega) /[\hbar k(E) \tau(E)]$ has been hidden for clarity, then

$$
\begin{aligned}
M_{n}\left(k_{\perp}\right) \approx & \frac{1}{8 \pi^{2}}\left(\frac{2 m}{\hbar^{2}}\right)^{(n+3) / 2} \int_{\mu+\phi}^{\mu+\hbar \omega} s^{n / 2}(s-\hbar \omega)^{1 / 2} \\
& \times \eta_{n}\left(\sqrt{\frac{\mu+\phi}{s}}\right) d s,
\end{aligned}
$$

where $p$ is a function of energy, and therefore a function of $s$. Electron-electron relaxation times in metals (which dominate for photoexcited electrons) are well approximated by a power-law relation $\tau_{e e}(E) \approx \tau_{o}[\mu /(E-\mu)]^{j}$ (e.g., Refs. [3,5]), where $\tau_{o}=1.23$ fs and $j=1.9$ for copper (or 3.44 fs and 1.85, respectively, for silver), suggesting that if $\delta(\omega)=12 \mathrm{~nm}$, then

$$
p(E) \approx 6.22\left(\frac{\mu}{E}\right)^{1 / 2}\left(\frac{E}{\mu}-1\right)^{1.9} .
$$

In particular, $\left.p(\mu+\Phi)\right|_{\mathrm{Cu}} \approx 2.10$. Therefore, when step functions are used to approximate the Fermi-Dirac distribution and the transmission probability, emittance takes the form

$$
\varepsilon_{n, \mathrm{rms}}=\frac{\rho_{c}}{2} \frac{1}{m c^{2}}\left\{\frac{\int_{\mu+\phi}^{\mu+\hbar \omega} s(s-\hbar \omega)^{1 / 2} \eta_{2}\left(\sqrt{\frac{\mu+\phi}{s}}\right) d s}{\int_{\mu+\phi}^{\mu+\hbar \omega}(s-\hbar \omega)^{1 / 2} \eta_{0}\left(\sqrt{\frac{\mu+\phi}{s}}\right) d s}\right\}^{1 / 2} .
$$

Explicit evaluation shows that the integrands of the numerator and denominator of Eq. (10) are smoothly varying: to a good approximation the integrand of the numerator behaves as a quadratic, and that of the denominator as a linear, function in $s$, and in both cases, the integrands evaluated at the lower bounds vanish as a consequence of $\eta_{n}(1)=0$. Using the linear and quadratic approximations in the denominator and numerator, respectively, the integrals can be trivially evaluated and are expressible in terms of the integrands evaluated at the upper bounds to give

$$
\frac{\varepsilon_{n, \mathrm{rms}}}{\varepsilon_{n, \mathrm{rms}}^{0}}=\frac{2 \eta_{2}(\Delta)}{\left(1-\Delta^{2}\right) \eta_{0}(\Delta)}
$$

where $\Delta=[(\mu+\phi) /(\mu+\hbar \omega)]^{1 / 2}$ has been introduced, the square of which is equal to the ratio of the height of the barrier to the energy of an electron excited from the Fermi level. When $\Delta=1$, the right-hand side (RHS) of Eq. (11) is identically unity, and is understood to be the asymptotic, or DS, approximation. However, to a good approximation, the right-hand side of Eq. (11) is close to unity over a range of $\Delta$, indicating that the DS approximation is good even when, first, the photon energy is not necessarily close to the height of the barrier above the Fermi level and, second, the energy dependence of the relaxation time is accounted for. The behavior is shown in Fig. 1 for typical copper parameters (albeit that the laser penetration depth factor $\delta_{\omega}$ is assumed fixed at $12 \mathrm{~nm}$ ) using the energy-dependent relaxation time for both a clean $(\Phi=4.5 \mathrm{eV})$ and partially cesiated $(\Phi=1.8 \mathrm{eV})$ surface. While a range of $\Delta$ values are shown, in practice, $\Delta$ is close to unity as laser wavelengths longer than UV are generally used [e.g., for a wavelength of $240 \mathrm{~nm}, \Delta(\mathrm{Cu})=0.972$ and $\Delta(\mathrm{Cs}-\mathrm{Cu})=$ 0.850].

A comparison of the DS approximation to measurements of the emittance of copper are definitively examined 


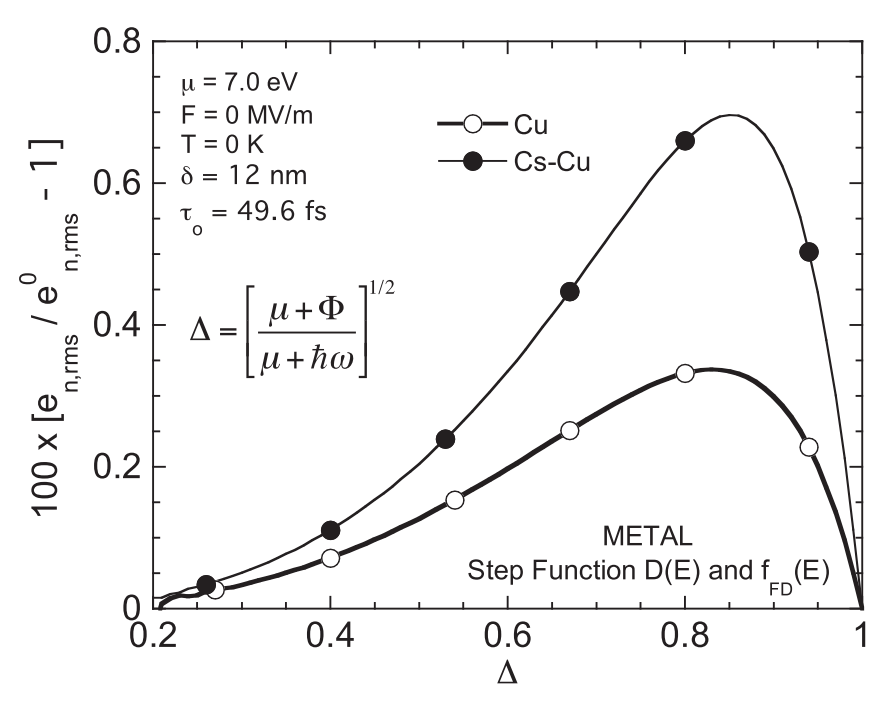

FIG. 1. Behavior of the ratio of emittance approximations, given in Eq. (11), for copper parameters.

by Dowell and Schmerge $[1,2,8]$, who compare their formulation with other treatments in the literature and provide a brief discussion of potential causes of differences between theory and experiment for metals, such as neglect of polarization effects, surface roughness, emission from a tilted surface, effects of field orientation, and field enhancement effects. We would augment this discussion by observing that crystal faces with their attendant work function differences and the nontrivial impact of adsorbates would tend to increase theoretical emittance estimates: patch fields created close to the photocathode surface create transverse field components. A comparable effect alluded to by Dowell and Schmerge concerning surface roughness and field enhancement is that electrons emitted from such surfaces are launched at their creation with velocity components parallel to the surface. In all, such factors increase photocathode "intrinsic" emittance from the start, and models that neglect such factors therefore underestimate the emittance by an amount difficult to anticipate. Simple models may provide qualitative estimates (and are underway).

\section{FIRST ORDER TEMPERATURE AND FIELD CORRECTIONS}

The step-function approximations to the transmission probability and the Fermi-Dirac distributions are now relaxed, and the consequences on $\varepsilon_{n, \text { rms }}$ assessed. To do so, introduce a function $f_{\varepsilon}(x)$, where $\varepsilon$ is a small parameter, of the form

$$
f_{\varepsilon}(x) \equiv\left(1+e^{\left(x-x_{o}\right) / \varepsilon}\right)^{-1} .
$$

Clearly, in the limit $\varepsilon \rightarrow 0, f_{\varepsilon}(-x)$ becomes a step function ( 0 for $x<x_{o}$ and 1 for $x>x_{o}$ ). For smoothly and slowly varying functions $h(x)$, the relation

$$
\int_{b}^{a} h(x) f_{\varepsilon}(x) d x \approx \int_{x_{o}}^{a} h(x) d x+\frac{1}{6}(\pi \varepsilon)^{2} h^{\prime}(x),
$$

where the upper and lower bounds satisfy $\left(a-x_{o}\right) / \varepsilon \gg 1$ and $\left(x_{o}-b\right) / \varepsilon \gg 1$ and $h^{\prime}(x)=d h / d x$. Because both the Fermi-Dirac distribution for temperature and the transmission probability for field give rise to functions of the form of Eq. (12), small parameters $\varepsilon_{T}$ and $\varepsilon_{F}$ arise, and so to leading order, the moments are

$$
M_{n}\left(k_{\perp}\right) \approx M_{n}^{0}\left(k_{\perp}\right)+\delta M_{n}^{F}+\delta M_{n}^{T},
$$

where the 0 superscript denotes the step-function approximation, where $F$ and $T$ in the superscripts denote field and temperature, respectively, and where terms of order $\left(\varepsilon_{F} \varepsilon_{T}\right)^{2}, \varepsilon_{F}^{4}, \varepsilon_{T}^{4}$, and smaller are neglected. Application of Eq. (13) to Eq. (3) gives rise to approximations of $\delta M_{n}^{F}$ and $\delta M_{n}^{T}$.

For the Fermi-Dirac distribution, $\varepsilon_{T}=1 / \beta_{T}=k_{B} T$, $x_{o}=\mu$, and $f_{\varepsilon_{T}}\left(x_{o}\right) \leftrightarrow 1-f_{\mathrm{FD}}(E)$. It follows

$$
\begin{aligned}
\delta M_{n}^{T}= & -\frac{\left(k_{B} T\right)^{2}}{96 k_{F}}\left(\frac{2 m}{\hbar^{2}}\right)^{(n+4) / 2}(\mu+\hbar \omega)^{(n-2) / 2} \\
& \times[(n+1) \mu+\hbar \omega] \eta_{n}(\Delta),
\end{aligned}
$$

where $\hbar k_{F}=\sqrt{2 m \mu}$, or, alternately,

$$
\begin{aligned}
\frac{\delta M_{2}^{T}}{M_{2}^{0}} & =-\frac{(3 \mu+\hbar \omega)\left(\pi k_{B} T\right)^{2}}{4 \mu(\mu+\hbar \omega)(\hbar \omega-\phi)} \\
\frac{\delta M_{0}^{T}}{M_{0}^{0}} & =-\frac{\left(\pi k_{B} T\right)^{2}}{6 \mu(\hbar \omega-\phi)},
\end{aligned}
$$

where the quadratic and linear approximations for the stepfunction approximations to $M_{2}$ and $M_{0}$, respectively, have been used in the denominators. For copper parameters at an elevated $T=600 \mathrm{~K}$ and fields of $100 \mathrm{MV} / \mathrm{m}$, the ratios are $\delta M_{2}^{T} / M_{2}^{0}=-0.0019$ and $\delta M_{0}^{T} / M_{0}^{0}=-0.0006$, and, therefore, the insensitivity of the DS approximation to temperature is reasonable.

To extend such an analysis to the impact of fields, we first note that, for a number of potential barriers encountered in electron emission, the transmission probability can be well approximated by $f_{\varepsilon}(x)$ [9]. Barriers for which this is true include the image charge and quadratic barrier potentials when they are described using the Kemble approximation to the transmission probability, but with the additional modification that the argument of the exponential (the "theta" or "area under the curve" term) is linear in energy $[4,5,10,11]$. The forms of $\varepsilon_{F}$ and $x_{o}$ require greater examination, but will require the evaluation of $\beta_{F}$ which in turn depends on whether tunneling or over-thebarrier emission dominates [10]: if the latter is assumed, then $\beta_{F} \approx(\pi / \hbar) \sqrt{2 m}\left(Q / F^{3}\right)^{1 / 4}$, where the terms, their definitions, and their values are summarized in Table I. However, unlike the case for temperature, the behavior of transmission probability ties the limits of the angular integration to the value of the energy, and therefore, deriva- 
tives with respect to the scattering factor arise, making the application of Eq. (13) require greater scrutiny. Making a change of integration variables to $s=E-\mu$ and $x=$ $\cos ^{2} \theta$, Eq. (3) becomes

$$
\begin{aligned}
M_{n}\left(k_{\perp}\right)= & \frac{1}{8 \pi^{2}}\left(\frac{2 m}{\hbar^{2}}\right)^{(n+3) / 2} \int_{-\mu}^{\infty} d s \sqrt{s+\mu} \\
& \times(s+\mu+\hbar \omega)^{n / 2} f_{\varepsilon_{T}}(s) f_{\varepsilon_{T}}(-s-\hbar \omega) \\
& \times \int_{0}^{1} \frac{(1-x)^{n / 2}}{2\left[x^{1 / 2}+p(s+\mu+\hbar \omega)\right]} \\
& \times f_{\varepsilon_{F}}\left[x_{o}(s)-x\right] d x,
\end{aligned}
$$

where again $s$ is a dummy integration variable not to be confused with similar usage in Eq. (4). It is deduced that

$$
\varepsilon_{F}(s)=\frac{1}{\beta_{F}(s+\mu+\hbar \omega)} \quad x_{o}(s)=\frac{\mu+\phi}{s+\mu+\phi} .
$$

Because terms of order $\left(\varepsilon_{F} \varepsilon_{T}\right)^{2}$ and higher are neglected to evaluate $\delta M_{n}^{F}$, the thermal terms in Eq. (17) are replaced with step functions that simply alter the limits of integration for $s$. It follows

$$
\begin{aligned}
\delta M_{n}^{F}= & \frac{1}{8 \pi^{2}}\left(\frac{2 m}{\hbar^{2}}\right)^{(n+3) / 2} \int_{-(\hbar \omega-\phi)}^{0} d s \sqrt{s+\mu} \\
& \times(s+\mu+\hbar \omega)^{n / 2} \frac{1}{6}\left[\pi \varepsilon_{F}(s)\right]^{2} h_{n}^{\prime}(s) d s,
\end{aligned}
$$

where $h_{n}^{\prime}(s)$ is the $x$ derivative of the coefficient of $f_{\varepsilon_{F}}$ in Eq. (17) evaluated at $x=x_{o}(s)$. The values of $s$ tend to be smaller than the additive terms with which $s$ appears, and so the integral of Eq. (19) may be approximated by the product of the integrand at its upper limit with the width of the integration region, or

$$
\begin{aligned}
\delta M_{n}^{F} \approx & \frac{1}{48}\left[\varepsilon_{F}(0)\right]^{2}\left(\frac{2 m}{\hbar^{2}}\right)^{(n+3) / 2}(\hbar \omega-\phi) \\
& \times \sqrt{\mu}(\mu+\hbar \omega)^{n / 2} h_{n}^{\prime}(0) .
\end{aligned}
$$

The general form for $n$ is cumbersome, and so the explicit forms for $n=2$ and 0 are given instead, where use has been made of $x_{o}(0)=\Delta^{2}$ and $p_{o}=p(\mu+\hbar \omega)$, from which

$$
\begin{aligned}
& \delta M_{2}^{F} \approx \frac{1}{192 \beta_{F}^{2}}\left(\frac{2 m}{\hbar^{2}}\right)^{5 / 2} \frac{\mu^{1 / 2}\left(1-\Delta^{2}\right)\left(1+2 p_{o} \Delta+\Delta^{2}\right)}{\Delta\left(p_{o}+\Delta\right)^{2}} \\
& \delta M_{0}^{F} \approx \frac{1}{192 \beta_{F}^{2}}\left(\frac{2 m}{\hbar^{2}}\right)^{3 / 2} \frac{\mu^{1 / 2} \Delta\left(1-\Delta^{2}\right)}{(\mu+\phi)\left(p_{o}+\Delta\right)^{2}} .
\end{aligned}
$$

Concise ratios with the zeroth order moments are not available because common factors which canceled in the ratios for the thermal case do not arise here. Direct numerical evaluation using copper parameters, a wavelength of $240 \mathrm{~nm}$, and a field of $100 \mathrm{MV} / \mathrm{m}$, for which $\Delta=$ $0.956, p_{o}=2.65$, and $\beta_{F}=70.1$, show that $\delta M_{2}^{F} / M_{2}^{0} \approx$
0.0019 and $\delta M_{0}^{F} / M_{0}^{0} \approx 7.7 \times 10^{-6}$. It follows that, as for temperature before, the moments are relatively insensitive to field effects apart from the Schottky barrier lowering factor.

\section{EXTENSION TO SEMICONDUCTORS}

The Moments formulation applied to positive electron affinity (PEA) semiconductors differs from that of metals because: the barrier at the surface is small making the transmission probability different than a step function; the distribution of photoexcited electrons differs; effective mass considerations arise; and band bending occurs. Doped semiconductors experience, like metals, an image charge potential modified barrier [12-14] in which the image charge term $Q$ is multiplied by a factor $\left(K_{s}-\right.$ $1) /\left(K_{s}+1\right)$, where $K_{s}$ is the static dielectric constant (e.g., for $K_{s}=5$, the factor is $2 / 3$ and the Schottky lowering factor therefore $\sqrt{2 / 3}$ of what it would be for a metal). If semiconductors are not sufficiently doped, then the effects on image charge and band bending are of greater complexity - the image charge appearing, for example, more triangular. If $n$-type layers are atop $p$-type bulk material (e.g., $\mathrm{Cs}_{3} \mathrm{Sb}$ on $\mathrm{Na}_{2} \mathrm{KSb}$ as in Fig. 6 of Ref. [15]), then the potential can be elaborate. Narrow triangular barriers can exist at the surface due to $\mathrm{CsO}$ even for negative electron affinity photocathodes [16]. The smallness of the PEA barrier is such that the adequacy of the Kemble approximation and a dielectric modified $\beta_{F}$ should not be presumed. While undeniably important, such issues are quite outside the present focus, and a model which allows us to divorce the two is desired. We take a cue from the observation that, for external fields on the order of $1 \mathrm{MV} / \mathrm{m}$, the barrier visually resembles a triangular barrier (albeit with a rounded apex), and that "triangular" permeates the discussion in general. Given that the transmission probability for the triangular barrier is well approximated analytically for arbitrary field for energies above the barrier maximum [17], the simplicity of the resulting theory commends its usage, as the impact of field is easy to accommodate even when the barrier height is small. Parenthetically, the triangular transmission probability in the zero field limit is the same as for a wide rectangular barrier of the same height [4], the form of which is better suited to the linearized Kemble approximation, but the use of a rectangular barrier transmission probability would require the adjustment of both height and width to mimic field effects, and therefore its utility is less. The triangular approximation will allow us to judge the relative impact of field effects compared to a zeroth order approximation, even as we note that the zeroth order approximation will intuitively be different, perhaps larger, than it would if the transmission probability were evaluated numerically from Schrödinger's equation.

The Moments formulation for semiconductors, given previously [18], is 


$$
\begin{aligned}
M_{n}\left(k_{\perp}\right)= & (2 \pi)^{-3} \int_{0}^{k_{\max }} 2 \pi k^{2} d k \int_{0}^{\pi / 2}\left(k_{\perp}^{n}\right) \\
& \times \sin \theta f_{\lambda}\left(\cos \theta, E_{s}\right) D\left(k_{z}\right) d \theta,
\end{aligned}
$$

where $k$ is measured with respect to the conduction band minimum, $k_{z}=k \cos \theta$, and $E_{s}$ is the energy of the electron inside the semiconductor $\left(\hbar^{2} k^{2} / 2 m^{*}\right)$ wherein the electron effective mass $m^{*}$ can be different than $m\left(m^{*}=0.118 m\right.$ for $\mathrm{Cs}_{3} \mathrm{Sb}$ as calculated using the alpha semiconductor model $[18,19])$. In contrast to the procedure in metals, where the energy $E_{k}$ in Eq. (3) is prior to the absorption of a photon, the $k$ terms in Eq. (22) are after photon absorption - and this reflects differences in the formulations because the conduction band of $p$-type semiconductors is free of carriers which would otherwise affect final state occupation factors giving rise to Fermi-Dirac functions present in the metal formulation. Rather, for semiconductors, such concerns are accounted for by using $k_{\max }$, (the maximum photoexcited electron energy) given by $\hbar^{2} k_{\max }^{2} / 2 m=\hbar \omega-E_{g}$. The scattering factor $f_{\lambda}$, referring as it does to transport within the semiconductor, requires $m^{*}$ : this shall be implicitly included in the $p\left(E_{s}\right)$ factor but, as shall be seen below and as anticipated by the metal formulation, the final result does not depend greatly on $p$. Therefore, in the vacuum,

$$
\begin{aligned}
M_{n}\left(k_{\perp}\right) \approx & \frac{1}{16 \pi^{2}}\left(\frac{2 m}{\hbar^{2}}\right)^{(n+3) / 2} \int_{0}^{\hbar \omega-E_{a}-E_{g}} d s \sqrt{s+E_{a}} \\
& \times\left(s+\hbar \omega+E_{a}\right)^{n / 2} \int_{x_{o}(s)}^{1}(1-x)^{n / 2} \\
& \times \frac{1}{x^{1 / 2}+p\left(s+E_{a}\right)} D\left[\left(s+E_{a}\right) x\right] d x,
\end{aligned}
$$

where $E_{a}$ and $E_{g}$ are the electron affinity and band gap, respectively, $x_{o}$ is redefined as $x_{o}(s)=E_{a} /\left(s+E_{a}\right)$, and to zeroth order, the transmission probability may be approximated by its zero field limit [see Eq. (205) of Ref. [4] or Eq. (10) of Ref. [18] ]

$$
D\left(E>E_{a}\right) \approx \frac{4\left[E\left(E-E_{a}\right)\right]^{1 / 2}}{\left[E^{1 / 2}+\left(E-E_{a}\right)^{1 / 2}\right]^{2}} .
$$

As with metals, the nonzero field extension of Eq. (24) results in a small contribution that primarily affects electrons near the conduction band minimum, and therefore, it will not contribute significantly unless the fields are substantial. Therefore, as with the DS approximation, the zeroth order approximation to the Moments for the evaluation of semiconductor photocathode emittance is adequate, albeit that the behavior of the scattering factor and the transmission probability change the analysis.

The smallness of the electron affinity $E_{a}(0.3 \mathrm{eV}$ for $\mathrm{Cs}_{3} \mathrm{Sb}$ ) undercuts accurate analytical approximations to Eq. (23): therefore, it shall be evaluated numerically. However, a DS-like approximation is instructive, in which the transmission probability is replaced by a step function and a trapezoidal approximation is made for the energy integral for all moments, albeit that in contrast to metals, the behavior of the integrand is not as well characterized by a polynomial, and so the trapezoidal approximation will not be as accurate. We find

$$
\begin{aligned}
M_{n}^{0}\left(k_{\perp}\right)= & \left(16 \pi^{2}\right)^{-1} E_{a}^{3 / 2}\left(\frac{2 m}{\hbar^{2}}\right)^{(n+3) / 2} \Delta_{s}^{-(n+3)} \\
& \times\left(2 E_{a}+E_{g} \Delta_{s}^{2}\right)^{n / 2}\left(1-\Delta_{s}^{2}\right) \eta_{n}\left(\Delta_{s}\right),
\end{aligned}
$$

where $\Delta_{s}=\left[E_{a} /\left(\hbar \omega-E_{g}\right)\right]^{1 / 2}$ and is not necessarily close to unity. Therefore, the DS-like approximation for emittance from semiconductors is

$$
\varepsilon_{n, \text { rms }}^{0}(\text { semi })=\frac{\rho_{c}}{2}\left\{\frac{\left(2 \hbar \omega-E_{g}\right)}{m c^{2}} \frac{\eta_{2}\left(\Delta_{s}\right)}{\eta_{0}\left(\Delta_{s}\right)}\right\}^{1 / 2} .
$$

This may be simplified by using $\eta_{2}\left(\Delta_{s}\right) / \eta_{0}\left(\Delta_{s}\right) \approx(1-$ $\left.\Delta_{s}^{2}\right) / 2$ (as for metals) to give

$\varepsilon_{n, \mathrm{rms}}^{0}(\mathrm{semi}) \approx \frac{\rho_{c}}{2}\left\{\frac{1}{2 m c^{2}} \frac{\left(2 \hbar \omega-E_{g}\right)}{\left(\hbar \omega-E_{g}\right)}\left(\hbar \omega-E_{g}-E_{a}\right)\right\}^{1 / 2}$.

The evaluation of emittance requires the dependence of $p(E)$ for semiconductors, though in practice its determination may be difficult and its representation by a power law or polynomial of a simplicity comparable to metals is unlikely due to the dependencies on the optical parameters and the scattering rates involved. However, for the purposes of comparing the numerical quality of the approximations, only a representative value $p(E)$ is required (e.g., analogous to the factor $p_{o}$ for metals): approximating $p(E)$ by a constant is sufficient to determine the adequacy of Eqs. (26) and (27) in approximating Eq. (23), and shall be how the approximations are judged. Two instances of semiconductor parameters shall be considered: the first shall be for $\mathrm{Cs}_{3} \mathrm{Sb}$, the second for $\mathrm{Cs}_{2} \mathrm{Te}$ parameters (a candidate that has generated interest in the literature [20,21]) suggested by Flöttman [22] and Sertore et al. [23]. In both cases, three different constant values of $p$ shall be considered, reflecting the wide differences semiconductors show for penetration depth and mean-free paths.

For $\mathrm{Cs}_{3} \mathrm{Sb}$, as per Ref. [18], let $\lambda=532 \mathrm{~nm}$, and $\rho_{c}=$ $0.1 \mathrm{~cm}$ and otherwise assume Table I parameters. For $p=$ $0.1,0.5$, and 1.0, then Eq. (23) predicts $\varepsilon_{n, \mathrm{rms}}=0.577$, 0.572 , and $0.568 \mathrm{~mm} \mathrm{mrad}$, whereas Eq. (26) predicts $0.686,0.679$, and $0.675 \mathrm{mmmrad}$, respectively, and Eq. (27) (which is independent of $p$ ) predicts $0.664 \mathrm{~mm}$ mrad. Therefore, although reasonable, the DS-like analogue for semiconductors shows greater differences (on the order of 20\%) from the numerical evaluation compared to metals.

For $\mathrm{Cs}_{2} \mathrm{Te}$, both Flöttman [22] and Sertore et al. [23] use the approximation $\varepsilon_{\text {flöt }}^{0}=\left(\rho_{c} / 2\right)\left[2 E_{\text {kin }} / 3 m c^{2}\right]^{1 / 2}$. As Flöttman argues, $E_{\text {kin }}$ is similar to, albeit not identical 
with, $\hbar \omega-E_{g}-E_{a}$. Assuming that relation anyway, Flöttman finds that a $\mathrm{Cs}_{2} \mathrm{Te}$ photocathode with a radius of $0.15 \mathrm{~cm}$ and with $E_{g}=3.5 \mathrm{eV}, E_{a}=0.2 \mathrm{eV}$ and a photon energy satisfying $\hbar \omega-E_{g}-E_{a}=0.55 \mathrm{eV}$ (i.e., $\lambda=292 \mathrm{~nm}$ ), that $\varepsilon_{\text {flöt }}^{0}=0.635 \mathrm{~mm}$ mrad. Similarly, Sertore et al., finds that, with a radius of $0.1 \mathrm{~cm}$ at a wavelength of $\lambda=211 \mathrm{~nm}$, then $\varepsilon_{\text {flöt }}^{0}=0.84 \mathrm{~mm} \mathrm{mrad}$, which compares well with their experimental measurement of $0.7 \pm 1 \mathrm{~mm}$ mrad. For $\mathrm{Cs}_{2} \mathrm{Te}$ we shall use the parameters $E_{g}=3.5 \mathrm{eV}, E_{a}=0.2 \mathrm{eV}, \lambda=211 \mathrm{~nm}$, and $\rho_{c}=$ $0.1 \mathrm{~cm}$. For $p=0.1,0.5$, and 1.0, then Eq. (23) predicts $\varepsilon_{n, \mathrm{rms}}=1.31,1.28$, and $1.27 \mathrm{~mm} \mathrm{mrad,} \mathrm{whereas} \mathrm{Eq.} \mathrm{(26)}$ predicts $1.46,1.42$, and $1.40 \mathrm{~mm} \mathrm{mrad}$, respectively, and Eq. (27) (which is independent of $p$ ) predicts $1.36 \mathrm{~mm}$ mrad.

While the approximate DS-like formulas are reasonable compared to the numerical evaluation, clearly the behavior of the integrands in the Moments matter, and for semiconductors, the approximations are more consequential when comparing to experiment. Differences between theory and measurement may include: rounding the potential barrier as per the image charge approximation; presence of nonzero transmission probabilities for tunneling below the barrier maximum; dependencies of $p(E)$ in the moments evaluation on optical penetration depth and the electron energy dependence of the scattering rates; effects of band bending when fields are present; presence of higher electron affinities (as anticipated by Flöttman) caused by adsorbates or the impact of patchy areas of emission on the surface; and uncertainties in the experimental measurements. All will have an impact, the determination of which requires a comprehensive numerical study, but it is equally clear that, in the face of such uncertainties, the closeness of theoretical estimates herein to the experimental values cited is (we argue) notable and comparable to metal theory-experiment differences.

For effects due to temperature, consider the $\mathrm{Cs}_{3} \mathrm{Sb}$ case in greater detail. The ratio of the emittance calculated using Eq. (25) is compared with the leading order Eq. (26) and shown in Fig. 2. For semiconductors, in particular, the laser penetration depth and scattering rates are complex functions of wavelength and energy: therefore, for the comparison in Fig. 2 and as done previously, only representative and constant values of $p$ were considered. Observe that the relative insensitivity to the precise value of $p$ could have been anticipated from metals: the general goodness of the "forward" approximation, in which $p+x^{1 / 2} \approx p+1$ in the denominator of the $x$ integral in Eq. (23), means that ratios of Moments cause the $(1+p)$ factors to cancel and render the result insensitive to $p$. Therefore, temperature effects, which would make their presence known via changes to values of the relaxation times, are expected to be small.

For differences due to field, the transmission probability below the barrier maximum has an exponen-

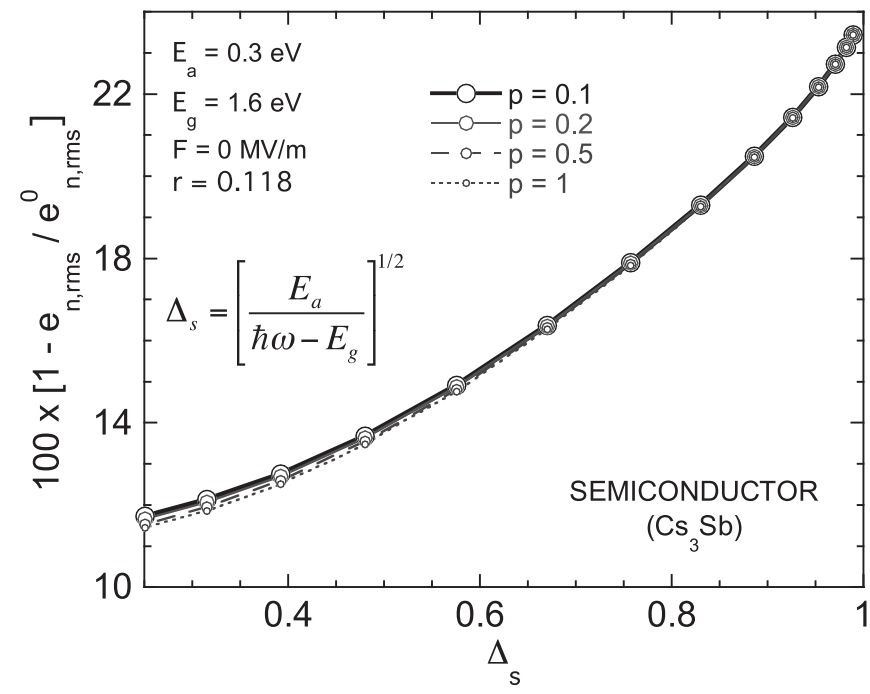

FIG. 2. Behavior of the ratio of emittance calculated numerically from Eq. (23) with its leading order trapezoidal approximation given by Eq. (26) for cesium antimonide parameters for various values of $p \approx$ constant.

tially decaying behavior so that, as with metals, the Moments should increase when nonzero fields are encountered. However, a detailed examination of the triangular barrier potential $[17,24]$ shows that the effects of field are governed by a field-dependent energy parameter given by $\left(4 \hbar^{2} F^{2} / 125 m\right)^{1 / 3}$ : even for fields comparable to $100 \mathrm{MV} / \mathrm{m}$, this energy parameter is of the size $0.007 \mathrm{eV}$, and therefore the emittance from a semiconductor, as with the DS approximation before it, is expected to be relatively insensitive to field corrections apart from Schottky barrier lowering, a conclusion supported by numerical studies [25].

\section{SUMMARY}

A derivation of the emittance of metal and semiconductor photocathodes using the Moments approach was given. The contributions of both temperature and field-dependent effects via changes to the Fermi-Dirac distribution governing electron statistics in a metal, or the transmission probability of a triangular barrier as for a semiconductor, were found. It has been demonstrated that the Dowell-Schmerge (DS) asymptotic expression for the emittance of a metal photocathode is better over a wider range of incident photon energies than its derivation would imply and remains excellent even when temperature and field effects are included. An asymptotic expression was derived for semiconductors and shown to have analogous features to the DS approximation for metals, but which is modified to a greater extent by the behavior of the transmission probability, and which tends to be less accurate compared to numerical results than for the case of metals. The derived formulas were applied to calculate the emittance of copper, 
cesium antimonide, and cesium telluride photocathodes as representative cases.

\section{ACKNOWLEDGMENTS}

We thank the Joint Technology Office and the Office of Naval Research for supporting this work. We are especially grateful to D. Dowell and J. Schmerge for bringing to our attention the necessity of including the photon energy in the evaluation of $\left\langle k_{\perp}^{2}\right\rangle$, and to D. Dowell in particular for many profitable discussions thereafter.

[1] D. H. Dowell and J.F. Schmerge, Phys. Rev. ST Accel. Beams 12, 074201 (2009).

[2] J. F. Schmerge, J. E. Clendenin, D. H. Dowell, and S. M. Gierman, Int. J. Mod. Phys. A 22, 4069 (2007).

[3] K. L. Jensen, P. G. O'Shea, D. W. Feldman, and N. A. Moody, Appl. Phys. Lett. 89, 224103 (2006).

[4] K. L. Jensen, Advances in Imaging and Electron Physics, Electron Emission Physics Vol. 149 (Academic Press, San Diego, 2007).

[5] K. L. Jensen, N. A. Moody, D. W. Feldman, E. J. Montgomery, and P.G. O'Shea, J. Appl. Phys. 102, 074902 (2007).

[6] P. G. O'Shea, Phys. Rev. E 57, 1081 (1998).

[7] K. L. Jensen, P. G. O'Shea, D. W. Feldman, and J. L. Shaw, J. Appl. Phys. 107, 014903 (2010).

[8] D. H. Dowell, F. K. King, R. E. Kirby, J. F. Schmerge, and J. M. Smedley, Phys. Rev. ST Accel. Beams 9, 063502 (2006).

[9] J. W. Gadzuk and E. W. Plummer, Phys. Rev. B 3, 2125 (1971).
[10] K. L. Jensen, J. Appl. Phys. 102, 024911 (2007).

[11] R. G. Forbes, J. Appl. Phys. 103, 114911 (2008).

[12] R. Stratton, Phys. Rev. 125, 67 (1962).

[13] E. H. Rhoderick, J. Phys. D 3, 1153 (1970).

[14] L. M. Baskin, O. I. Lvov, and G. N. Fursey, Phys. Status Solidi B 47, 49 (1971).

[15] B. Erjavec, Thin Solid Films 303, 4 (1997).

[16] G. Vergara, A. Herrera-Gomez, and W.E. Spicer, Surf. Sci. 436, 83 (1999).

[17] K. L. Jensen, J. Vac. Sci. Technol. B 21, 1528 (2003).

[18] K. L. Jensen, B. L. Jensen, E. J. Montgomery, D. W. Feldman, P. G. O'Shea, and N. Moody, J. Appl. Phys. 104, 044907 (2008).

[19] B. Jensen, and K. L. Jensen, J. Comput. Theor. Nanosci. 6, 1770 (2009).

[20] S. H. Kong, J. Kinrosswright, D. C. Nguyen, and R. L. Sheffield, J. Appl. Phys. 77, 6031 (1995).

[21] I. Boscolo and P. Michelato, Nucl. Instrum. Methods Phys. Res., Sect. A 445, 389 (2000).

[22] K. Flöttmann, TESLA FEL-Report No. 1997-01, 1997.

[23] D. Sertore, D. Favia, P. Michelato, L. Monaco, and P. Pierini, European Particle Accelerator Conference, Lucerne, Switzerland (2004), pp. 408-410, MOPKF045 [http://accelconf.web.cern.ch/AccelConf/e04/default. htm].

[24] K. L. Jensen, and E. J. Montgomery, J. Comput. Theor. Nanosci. 6, 1754 (2009).

[25] K. L. Jensen, D. W. Feldman, E. J. Montgomery, N. A. Moody, and J. Petillo, in Proceedings of the IEEE Particle Accelerator Conference (IEEE, New York, 2007), pp. 1206-1208, TUPMS091 [http://ieeexplore. ieee.org/stamp/stamp.jsp?tp $=\&$ arnumber $=4441031 \&$ isnumber $=4439905$ ]. 